\title{
Design of the Bracing in Adolescent Idiopathic Scoliosis Trial (BrAIST)
}

\author{
Stuart L. Weinstein, MD [Professor], \\ Department of Orthopaedics and Rehabilitation University of lowa \\ Lori A. Dolan, PhD [Associate Research Scientist], \\ Department of Orthopaedics and Rehabilitation University of lowa
}

James G. Wright, MD, MPH, FRCSC, and

Department of Surgery - The Hospital for Sick Children (Toronto, Ontario, Canada); the Division

of Orthopedic Surgery - University of Toronto (Toronto, Ontario, Canada)

\section{Matthew B. Dobbs, MD \\ Department of Orthopaedic Surgery Washington University School of Medicine and Saint Louis Shriners Hospital for Children}

\begin{abstract}
Adolescent idiopathic scoliosis (AIS) is characterized by a lateral curvature of the spine greater than 10 degrees with rotation of the spinal vertebrae. Two to three percent of children younger than 16 years of age will have a curvature of 10 degrees or more, but only 0.3-0.5 percent will require some sort of treatment. ${ }^{1}$ Progression of a spinal curve to 50 degrees suggests a high risk for continued curve progression throughout adulthood and usually indicates the need for spinal fusion surgery. Approximately 10 percent of adolescents with AIS end up having curves that progress to this degree; however, these 10 percent impose large costs in the US healthcare system. In 2009 there were over 3600 discharges for idiopathic scoliosis fusion surgery; the total costs of which ranked second only to appendicitis among children aged 10 to 17 ( $\$ 514$ million total; \$137 million to Medicaid alone). ${ }^{2}$
\end{abstract}

\section{Natural History of AIS}

Treatment of any condition is an attempt to alleviate current problematic signs and symptoms, and to ultimately alter long-term natural history. The vast majority of AIS patients do not initially present due to symptoms, but due to the finding of truncal asymmetry noted during screening or incidentally during well-child examinations. Few long-term studies exist, but they suggest AIS is primarily a spinal deformity associated with little significant physical or psychological disability, although the population may have a higher prevalence of back pain, and of respiratory compromise if the curve becomes extremely large. ${ }^{3-8}$ Therefore, the treatment of AIS during adolescence is mainly an attempt to prevent problems during adulthood by arresting the progression of the curve. Large

Correspondence: Stuart L. Weinstein, MD Department of Orthopaedics and Rehabilitation, University of Iowa 200 Hawkins Dr. Iowa City, Iowa 52242 (319) 356-1872 (319) 353-7919 (fax stuart-weinstein@uiowa.edu. 
curves can only be corrected through surgery. Thus, many patients seek and receive essentially prophylactic non-operative treatment for AIS.

\section{Bracing Treatment}

Treatment with rigid bracing (thoracolumbosacral and lumbosacral orthoses, TLSO, LSO) is the most common non-operative strategy to prevent curve progression. Many different designs exist, but all attempt to restore the normal contours and alignment of the spine through the use of external forces and, with some designs, the stimulation of active correction as the patient moves the spine away from pressures within the brace. Permanent correction of the curve is typically not expected instead the brace functions as a holding device during the high risk growth phase. Bracing is generally indicated for curves of greater than 20 degrees in adolescents who still have significant skeletal growth remaining. The recommended wear time varies across clinicians, ranging from 12 to 23 hours per day until skeletal maturity is reached ( $2-4$ years of treatment). Bracing, however, has many disadvantages for patients including the need for radiographs to monitor brace fit and curve response, out-of-pocket direct and indirect medical expenses, interference with sports and other activities, limited clothing choices, and self-consciousness about the brace. Brace wear for many patients is a constant reminder of their medical condition.

Over the past half century, many investigators have examined the effectiveness of bracing in AIS. ${ }^{9-69}$ The majority of studies have been uncontrolled case series or retrospective cohort studies, but conclusions from the few higher-level designs are limited by the lack of an untreated control group, ${ }^{37,66}$ randomized assignment, ${ }^{49}$ blinded outcome determination, ${ }^{37,49,66}$ and a priori determination of the necessary effect size. ${ }^{37,49,66}$ Therefore, the results of these studies yield inadequate evidence concerning the effectiveness of bracing.

When designing this trial, we sought to overcome the limitations of previous bracing studies. Therefore, we proposed a multi-center, randomized, controlled trial evaluating the effectiveness of bracing relative to watchful waiting in subjects with (AIS), using blinded, independent outcome measurement. The purpose of this manuscript is to outline the development and initiation of the Bracing in Adolescent Idiopathic Scoliosis Trial. Additional details are provided in the Appendix.

\section{Preliminary Work and Grant Funding}

Development of the science and infrastructure of the trial was supported by a Clinical Trial Planning Grant (R21-AR-49587) from the National Institutes of Arthritis and Musculoskeletal and Skin Diseases (NIAMS) of the National Institutes of Health (NIH). The BrAIST planning grant had four general goals: 1) appointing key personnel and creating the structural organization of the trial;2) developing procedures for data management and safety monitoring; 3) developing materials, methods and the data analysis plan; and 4) recruiting participating institutions. Beyond these goals, most resources during the planning grant phase were directed at demonstrating feasibility: establishing the ethics of randomization; ${ }^{70}$ estimating the willingness of adolescents and their parents to enroll into a randomized trial, and their preferences for treatment and their required benefit; ${ }^{71}$ and determining the 
reliability and validity of the brace wear monitoring system. ${ }^{72}$ Funding from the NIH/ NIAMS for the clinical trial was obtained in September 2006 (RO1-AR-O52113); support for central administration and clinical sites was also obtained through the Canadian Institute of Health Research (CIHR) the Shriners Hospitals for Children.

\section{Trial Organization}

Figure 1 is an overview of the major organizational components of BrAIST. The Executive Committee included the NIH Principal Investigator, the Clinical Trial Director, the PIs of the CIHR and Shrine grants, the director of the Data Management Center (DMC) and a representative from the NIAMS. Three major work groups resided at the University of Iowa. The Trial Director and the staff of the Clinical Coordinating Center (CCC) implemented the policies and procedures set by the PI and the Executive Committee, and coordinated the activities of the DMC, the Radiographic Reading Center (RRC) and the clinical sites.

\section{Study Design}

BrAIST is an innovation in AIS research because it combined components never included in a single study to date: simultaneous comparison of untreated and treated subjects; objective brace dose monitoring; comprehensive radiographic, clinical, and psychosocial testing; diversity of participating sites; blinded independent determinations of outcomes; and $a$ priori determination of effect size based on the risk/benefit considerations of potential patients.

BrAIST was planned and funded as a randomized study. After three years of data collection it became clear enrollment goals would not be reached within a reasonable time frame. Participating sites encountered significantly fewer eligible patients than estimated, and the percentage of patients agreeing to randomization was less than the $25 \%$ anticipated based on preliminary work. ${ }^{71}$ The primary reason reported for declining randomization was holding a strong preference for one treatment over the other. Therefore, in November 2009, BrAIST evolved from a completely randomized study to one including a preference arm. Patients who declined randomization could enter the trial by choosing either bracing or watchful waiting. Figure 2 summarizes the final trial protocol.

\section{Study Aims}

The primary aim of BrAIST was to compare the risk of curve progression to $\geq 50$ degrees (a proxy for surgical indication) in subjects treated by a brace to those treated by watchful waiting. Secondary aims include comparison between the health and functioning, quality of life, and self-image over time in the two treatment groups; determination of the relationship between bracing dose (wear time) and curve response; and development of a predictive model for curve progression based on individual patient characteristics at initial presentation (i.e., sex, skeletal maturity, chronological age), curve characteristics (i.e., curve magnitude, location), and treatment (bracing dose), and based on these characteristics, to estimate degree of risk reduction associated with the use of a brace. 


\section{Study Sites}

Data was collected at 25 participating sites (Table I) that began recruitment at varying times between March 2007 and January 2010. Four additional sites did not randomize any subjects within their first year of participation and were dismissed from the study.

\section{Study Population}

The target population for the BrAIST is patients with AIS at high risk for progression due to their age, skeletal immaturity and curve size. ${ }^{73-75}$ The inclusion criteria are listed in Table II. Patients who were previously braced or who had undergone scoliosis surgery were excluded. Subjects were also required to have insurance coverage or be willing to pay outof-pocket for their treatment.

\section{Recruitment and Enrollment}

Participating physicians assessed all patients presenting with AIS as part of routine clinical care and introduced the study to those meeting the inclusion criteria. To standardize the information given to patients and to minimize potentially biasing input from their physicians, coordinators, not physicians, were responsible for the education and consent processes. All patients who met the inclusion criteria were asked to read the online BrAIST Education Module (http://www.uiortho.com/braist/index2.htm). The Web-based module introduced the purpose of the trial, as well as the process of random treatment assignment vs. treatment selection, followed by presentation of the natural history of AIS and risk factors for progression, treatment options and a side-by-side comparison of bracing and watchful waiting. Advantages and disadvantages of each treatment were presented.

All eligible patients were registered by the coordinators on the DMC's Web-based enrollment system, which was available 24 hours per day. Those who declined participation were registered as "screened" and the following data were recorded: age, sex, ethnicity, race, SRS curve type, Cobb angle of the largest curve, and reason for declining participation. These data were used to compare participating with non-participating subjects to evaluate selection bias and representativeness of the sample to the population.

\section{Study Interventions}

Bracing Protocol-The trial was limited to the use of full-time, rigid TLSO's. Participating physicians and orthotists prescribed and fabricated the type of brace used during normal clinical practice; all were custom-fit using a plaster or fiber cast impression, measurements or via CAD-CAM systems software and completed with pads, slings, relief areas and other modifications. Braces were to be worn at least 18 hours per day. An in-brace standing posteroanterior (PA) spine radiograph was obtained at 4-6 weeks after brace delivery. Stowaway or TidbiT temperature loggers were embedded in the brace (Onset Computer Bourne, Massachusetts), and programmed to log the date, time and temperature every 15 minutes.

Watchful waiting-The subjects in this group underwent clinical and radiographic evaluation every 6 months, but were otherwise untreated. 
Change in Treatment Group-Subject-initiated treatment change (crossover) occurred when a subject stated they would not continue in the treatment they were originally assigned to (either by randomization or preference), i.e., when subject in the watchful waiting group requested a brace. This decision was to be made by the subjects and family and never suggested by the physician or coordinator. A subject who wore the brace less than prescribed was considered non-compliant, but still analyzed in the bracing arm.

Blinding - Neither the subject nor the physicians were blinded to treatment. However, the staff at the RRC, who were responsible for evaluating the radiographs and determining endpoints, were blinded to the treatment received.

\section{Data Collection and Follow-up Periods}

BrAIST collected radiographic, clinical, orthotic and self-report data at 6-month intervals. A complete list of these data is given in Table III.

Radiographic Data-Standing full spine PA and lateral films, supine side-bending (to assess curve flexibility) and left hand films for bone age were obtained at the initial visit. PA spine and hand films were obtained at each 6-month follow-up, with a lateral spine film taken yearly.

All films were uploaded to the RRC file transfer site and underwent extensive evaluation by a research associate and the study radiologist. Critical evaluations, such as the Cobb angle(s), Risser sign, apical vertebral rotation, kyphosis, lordosis and digital maturity stage, were arrived at via consensus between the two readers. Disagreements between the readers that exceeded certain pre-determined tolerance limits (Table IV) were resolved and the consensus measurement/classification was entered into the database.

Clinical Data-Subjects were seen by the clinician at each visit and underwent examination to rule out any neurological or other potential reason for the curvature. Chest, shoulder and back asymmetries were evaluated. The subjects' height was measured three times, and the average recorded.

Self-Report-All subjects completed the Child Health Questionnaire (CHQ), ${ }^{76}$ the PedsQL, ${ }^{77}$ the Self-Image Questionnaire for Young Adolescents (SIQYA), ${ }^{78}$ and the Spinal Appearance Questionnaire ${ }^{79}$ at each visit. Subjects in the bracing arm completed a 2-week brace wear diary between each follow-up visit.

Orthotic Evaluation (bracing arm only)—At the initial visit the orthotist recorded evaluations of the curve, coronal decompensation, shoulder and pelvis asymmetry, and described the type of brace to be fabricated (e.g., Boston, Wilmington) along with the specific customizations. At brace delivery and subsequent visits, the orthotist observed the subjects both in, and out, of the brace. Brace fit, curve correction and the condition of skin and bony prominences under the brace were recorded. Any additional modifications were recorded. Temperature monitor data (date, time stamps and temperature) were downloaded at least every 6 months by the research coordinator. Temperatures $\geq 82.4^{\circ} 72$ indicated that the brace was being worn. 


\section{Endpoints and Outcomes}

Subjects exited the study, and their outcome determined, when the first of two conditions was met: curve progression to $\geq 50$ degrees (treatment failure), or skeletal maturity (treatment success) as determined by consensus evaluation by the RRC. Thus, the length of time in the study differed across subjects. In the case when the two primary readers did not agree whether an endpoint had been met or not, a third, blinded reader was used to break the tie.

\section{Treatment Failure: Curve Progression to $\geq 50$ Degrees}

Cobb angle progression to 50 degrees or more has two major implications which make its use appropriate for this study. First, natural history studies have shown that curves greater than 50 degrees at skeletal maturity continue to progress throughout adulthood. Researchers have found greater continued progression in curves between 50 and 75 degrees at skeletal maturity relative to those of smaller magnitude. ${ }^{73}$ Secondly, curve progression beyond 50 degrees is commonly used as the threshold for considering surgical correction. This curve threshold indicates treatment failure and therefore has significant clinical implications as well as implications for the patient and family.

\section{Treatment Success: Skeletal Maturity}

The continued risk of curve progression is directly tied to amount of skeletal growth remaining. Therefore, reaching skeletal maturity with a Cobb angle less than 50 degrees indicated a treatment success. The original BrAIST maturity endpoint was based on change in vertical height adjusted for change in the Cobb angle. ${ }^{80}$ An adjusted change of $<1 \mathrm{~cm}$ over a 12 month period was defined as end of growth. In the course of the study, we found several instances where determining this endpoint was problematic (i.e. if study visits were missed or different stadiometers were used). Therefore, in 2011 with approval of the DSMB, the maturity endpoint was redefined as a Risser sign of 4 and a Sanders digital maturity stage (DMS) of 7 (Risser 5, DMS 7 for boys). ${ }^{81,82}$

\section{Statistical Analysis Plan}

Primary Aim-The primary analysis will estimate the adjusted odds ratio (OR) and 95\% confidence intervals of the association between bracing and a successful outcome (reaching skeletal maturity with a curve $<50$ degrees). Subjects from the randomized and preference arms will be combined and analyzed according to treatment received.

Propensity scores will be used to reduce the effect of treatment-selection bias (due to nonrandomized treatment assignment and/or cross-over) in the estimation of the treatment effect. ${ }^{83,84}$ Treatment success will be modeled as a function of treatment received, length of follow-up, and the propensity score. A minimally clinically significant difference in treatments will claimed if the relative risk reduction due to bracing is $\geq 50$ percent.

Interim analyses will be performed as requested by the DSMB. The cumulative Type I error rate will be maintained at the planned 0.05 by using the Lan and Demets ${ }^{85}$ spending function approach with the O'Brien-Fleming ${ }^{86}$ spending function. These methods adjust the 
threshold for statistical significance based on information time (the proportion of subjects with an endpoint relative to the number of subjects in the study population).

Sample Size and Power-The initial sample size calculations for BrAIST assumed randomization and equal number of subjects in each arm. The bracing failure rate was set at $15 \%$ based on the literature and the consensus of the protocol development committee. A survey of potential patients indicated that the minimally significant difference should be a $50 \%$ reduction in risk of curve progression to surgical indications, so the failure rate in the watchful waiting group was set at $30 \% .{ }^{71}$ For simplicity, it was assumed that a continuity corrected chi-square test would be used to compare the proportions. Setting alpha at 0.05 and power at $90 \%$, a sample of 348 subjects (384 after allowing for $10 \%$ loss-to-followup) was required.

Addition of the preference arm resulted in unequal numbers of subjects in each treatment group. The final proportions will not be known until the end of data collection and all crossovers between arms are accounted for. Assuming the proportion of subjects analyzed in the bracing arm ranges between 25 and $75 \%$ of the sample, the power of the test will be at least 80 percent. Table $\mathrm{V}$ provides a summary of these calculations.

\section{Secondary Aims}

\section{Health and Functioning}

The first secondary aim is to compare the CHQ, PedsQL, SIQYA and SAQ scores over time in the two treatment groups. Analysis will focus on comparing change in variables from baseline to study completion attributable to treatment using a linear mixed model approach.

\section{Dose-Response Relationship}

Our efforts will be directed to determining the smallest dose significantly associated with curve stabilization ( $<10$ degrees progression), as well as the maximum dose beyond which no further benefits are seen. We will first establish unadjusted dose response curves, then proceed to address the possibility of different curve response dependent upon patient and curve characteristics.

\section{Prediction of Curve Progression}

Curve progression will be defined in two ways: beyond 50 degrees (binary, yes or no) and as the difference between baseline and final follow-up (interval). Logistic and linear mixed models of curve progression will take into account patient and curve characteristics measured at initial presentation such as chronological age, Risser sign, menarche, sex, bodymass index, curve classification, Cobb angle, and other radiographic measures. The composite of brace dose, curve correction in brace, and change in vertebra wedging will then be added into the equations to model the risk of progression with treatment. This will allow estimation of risk for different clinical presentations, and to estimate how bracing may differentially alter the course of progression dependent on presentation. 


\section{Training and Ongoing Quality Control}

All procedures, measurements and data collection procedures were standardized and described in detail in the MOOP. In order to ensure quality data, the DMC and CCC developed valid and logically consistent case report forms, carefully trained those who were collecting and entering data, pilot-tested all forms and systems, carefully edited all data when it was entered, and monitored onsite to ensure that the data in the database was accurate.

\section{Participating Sites}

Training sessions were held for the investigators, site coordinators, site orthotists and funding organization representatives prior to trial initiation. At these meetings details of the trial were discussed, data collection questions clarified, and radiographic and orthotic standards reinforced. Prior to any enrollments, all coordinators were given several vignettes and required to go through the process of registering and enrolling simulated patients. Physicians were given a set of $\mathrm{x}$-rays which had been evaluated by the RRC staff and were required to complete their own evaluations and submit the case report forms. CCC staff held monthly phone calls with coordinators at each site to discuss each subject, including followup scheduling, data collection and submission and any issues concerning compliance, adverse events or disease progress or response. Yearly site monitoring visits were undertaken for the purpose of auditing records and making important site-specific recommendations to improve the overall trial quality. Coordinators attended yearly meetings organized by the CCC.

\section{Radiographic Reading Center}

The RRC consisted of a research assistant and a musculoskeletal radiologist, both of whom were trained specifically to perform the measurements and evaluations for the study using the standard definitions established by the Spinal Deformity Study Group and published in their manual. ${ }^{87} \mathrm{An}$ important consideration is that the endpoints (Cobb angle and skeletal maturity), were defined consistently, and measured with equal accuracy, across the treatment groups. This was ensured to a reasonable degree by the standards for $\mathrm{x}$-ray acquisition, blinding of the raters in the RRC, the software used in the RRC, and the ongoing quality assurance program built into the trial oversight.

\section{Data Management Center}

Once case report forms were received at the DMC, they were logged into a database, and queries emailed to the coordinators in the case of missing fields or other issues. Once resolved, the DMC staff double-data entered each form.

During the active enrollment period, the DMC produced monthly progress summarizing the recruitment and data entry to date. Similar progress reports were presented to the DSMB. These reports summarized current enrollment, follow-up data acquisition and database entry status, protocol deviations, and baseline characteristics of subjects in both treatment arms. 


\section{Data Safety and Monitoring Board (DSMB)}

BrAIST was a Phase III trial and oversight by a DSMB was required per NIH policy. The DSMB was composed of five members appointed by the NIAMS, all of whom were completely independent of the investigator and without conflicts of interest with the trial. Prior to the first enrollment, the DSMB reviewed and approved the protocol, informed consent documents and the safety monitoring and statistical analysis plan. During the course of data collection, the Board met approximately every 6 months to evaluate the progress of the trial, including recruitment, data quality and completeness, protocol violations and adverse events. All changes to the protocol, including the maturity endpoint and change from a completely randomized design were presented and approved by the Board.

\section{Conclusion}

BrAIST is an innovative effort which has enrolled the largest sample to date to study the effectiveness of bracing and key methodological improvements over past efforts have been incorporated. Clinical decision making will be improved by translation of the BrAIST results into evidence-based prognosis and estimates of how the prognosis, specifically the risk of progressing to surgery, may be altered by the use of bracing.

\section{Supplementary Material}

Refer to Web version on PubMed Central for supplementary material.

\section{Acknowledgement}

The authors would like to acknowledge the contributions of the BrAIST Protocol Development Committee: Keith Bridwell, MD, Robert Dickson, MD, Caroline Goldberg, MD, Jose Morcuende, MD, John Lonstein, MD, Raymond Morrissey, MD, Alf Nachemson, MD, Peter Newton, MD, and Dennis Wenger, MD; and the BrAIST Study Group: Mark Abel, MD, Oheneba Boachie-Adjei, MD, Jacques d'Astous, MD, John Emans, MD, John (Jack) Flynn, MD, Joseph Gerardi, DO, Daniel Green, MD, Kenneth Guidera, MD, Munish Gupta, MD, Kim Hammerberg, MD, M. Timothy Hresko, MD , Henry Iwinski, MD, Antony Kallur, MD, Walter Krengel, MD, Charles Mehlman, MD, Peter Newton, MD, Jean Ouellet, MD, Nigel Price, MD, Chris Reilly, MD, James O. Sanders, MD, Michael Schmitz, MD, Suken Shah, MD, and W. Timothy Ward, MD as well as previous members, Patrick Bosch, MD, Matthew Halsey, MD, Richard Schwend, MD, Kit Song, MD, Peter Sturm, MD and Elizabeth Szalay, MD.

The device(s)/drug(s) that is/are the subject of this manuscript is/are exempt from FDA or corresponding national regulations. The project described was supported by Award Numbers R21AR049587 and R01AR052113 from the National Institute of Arthritis and Musculoskeletal and Skin Diseases (Weinstein SL, PI); the Shriners Hospitals for Children (\#79125, Dobbs, MB, PI); the Canadian Institutes of Health Research (FRN-81050, Wright JG, PI); the University of Rochester (Sanders JO, PI); the Children's Mercy Hospital and Clinics (Price NJ, PI); and the Children's Miracle Network (Weinstein SL, PI). Relevant financial activities outside the submitted work: grants, board membership, royalties, travel/accommodations/meeting expenses, patents, consultancy.

\section{References}

1. Nachemson, AL.; Lonstein, JE.; Weinstein, SL. Scoliosis Research Society. Denver, Colorado: 1982. Report of the Prevalence and Natural History Committee of the Scoliosis Research Society..

2. HCUP Kids' Inpatient Database (KID). Healthcare Cost and Utilization Project (HCUP). Agency for Healthcare Research and Quality; Rockville, MD: 2009. www.hcup-us.ahrq.gov/kidoverview.jsp

3. Pehrsson K, Larsson S, Oden A, et al. Long-term follow-up of patients with untreated scoliosis. A study of mortality, causes of death, and symptoms. Spine. 1992; 17:1091-6. [PubMed: 1411763]

4. Weinstein SL, Dolan LA, Spratt KF, et al. Health and function of patients with untreated idiopathic scoliosis: a 50-year natural history study. JAMA. 2003; 289:559-67. [PubMed: 12578488] 
5. Ascani E, Bartolozzi P, Logroscino CA, et al. Natural history of untreated idiopathic scoliosis after skeletal maturity. Symposium on Epidemiology, Natural History and Non-operative Treatment of Idiopathic Scoliosis. Spine. 1986; 11:784-9. [PubMed: 3810293]

6. Goldberg MS, Mayo NE, Poitras B, et al. The Ste-Justine Adolescent Idiopathic Scoliosis Cohort Study. Part II: Perception of health, self and body image, and participation in physical activities. Spine. 1994; 19:1562-72. [PubMed: 7939992]

7. Mayo NE, Goldberg MS, Poitras B, et al. The Ste-Justine Adolescent Idiopathic Scoliosis Cohort Study. Part III: Back pain. Spine. 1994; 19:1573-81. [PubMed: 7939993]

8. Poitras B, Mayo NE, Goldberg MS, et al. The Ste-Justine Adolescent Idiopathic Scoliosis Cohort Study. Part IV: Surgical correction and back pain. Spine. 1994; 19:1582-8. [PubMed: 7939994]

9. Allington NJ, Bowen JR. Adolescent idiopathic scoliosis: treatment with the Wilmington brace. A comparison of full-time and part-time use. J Bone and Joint Surg. 1996; 78:1056-62. [PubMed: 8698723]

10. Andriacchi T, Schultz A, Belytschko T, et al. Milwaukee brace correction of idiopathic scoliosis. J Bone and Joint Surg. 1976; 58A:806-15. [PubMed: 956227]

11. Aulisa AG, Guzzanti V, Galli M, et al. Treatment of thoraco-lumbar curves in adolescent females affected by idiopathic scoliosis with a progressive action short brace (PASB): assessment of results according to the SRS committee on bracing and nonoperative management standardization criteria. Scoliosis. 2009; 4:21. [PubMed: 19765288]

12. Bassett G, Bunnell W, MacEwen G. Treatment of idiopathic scoliosis with the Wilmington brace. Results in patients with a twenty to thirty-nine degree curve. J Bone and Joint Surg. 1986; 68A: 602-5. [PubMed: 3957986]

13. Bassett GS, Bunnell WP. Influence of the Wilmington brace on spinal decompensation in adolescent idiopathic scoliosis. Clinical Orthopaedics \& Related Research. 1987:164-9. [PubMed: 3652570]

14. Brox JI, Lange JE, Gunderson RB, et al. Good brace compliance reduced curve progression and surgical rates in patients with idiopathic scoliosis. Eur Spine J. 2012; 21:1957-63. [PubMed: 22661234]

15. Bunnell WP. Nonoperative treatment of spinal deformity: the case for observation. Instructional Course Lectures. 1985; 34:106-9. [PubMed: 3833932]

16. Bunnell WP, MacEwen GD, Jayakumar S. The use of plastic jackets in the non-operative treatment of idiopathic scoliosis. J Bone and Joint Surg. 1980; 62A:31-8. [PubMed: 7351413]

17. Carr W, Moe J, Winter R, et al. Treatment of idiopathic scoliosis in the Milwaukee brace. J Bone and Joint Surg. 1980; 62A:599-612. [PubMed: 7380859]

18. Castro FP Jr. Adolescent idiopathic scoliosis, bracing, and the Hueter-Volkmann principle. Spine Journal: Official Journal of the North American Spine Society. 2003; 3:180-5.

19. Chase AP, Bader DL, Houghton GR. The biomechanical effectiveness of the Boston brace in the management of adolescent idiopathic scoliosis. Spine. 1989; 14:636-42. [PubMed: 2749381]

20. D'Amato CR, Griggs S, McCoy B. Nighttime bracing with the Providence brace in adolescent girls with idiopathic scoliosis. Spine. 2001; 26:2006-12. [PubMed: 11547201]

21. Danielsson AJ, Hasserius R, Ohlin A, et al. A prospective study of brace treatment versus observation alone in adolescent idiopathic scoliosis: a follow-up mean of 16 years after maturity. Spine. 2007; 32:2198-207. [PubMed: 17873811]

22. Edmonsson AS, Morris JT. Follow-up study of Milwaukee brace treatment in patients with idiopathic scoliosis. Clinical Orthopaedics \& Related Research. 1977:58-61. [PubMed: 598140]

23. Emans J, Kaelin A, Bancel P, et al. The Boston bracing system for idiopathic scoliosis. Follow-up results in 295 patients. Spine. 1986; 11:792-801. [PubMed: 3810295]

24. Federico DJ, Renshaw TS. Results of treatment of idiopathic scoliosis with the Charleston bending orthosis. Spine. 1990; 15:886-7. [PubMed: 2259975]

25. Fernandez-Feliberti R, Flynn J, Ramirez N, et al. Effectiveness of TLSO bracing in the conservative treatment of idiopathic scoliosis. Journal of Pediatric Orthopedics. 1995; 15:176-81. [PubMed: 7745089]

26. Focarile FA, Bonaldi A, Giarolo MA, et al. Effectiveness of nonsurgical treatment for idiopathic scoliosis. Overview of available evidence. Spine. 1991; 16:395-401. [PubMed: 1828625] 
27. Gammon SR, Mehlman CT, Chan W, et al. A comparison of thoracolumbosacral orthoses and SpineCor treatment of adolescent idiopathic scoliosis patients using the Scoliosis Research Society standardized criteria. J Pediatr Orthop. 2010; 30:531-8. [PubMed: 20733415]

28. Gardner ADH, Burwell RG, Wozniak AP, et al. Some beneficial effects of bracing and a search for prognostic indicators in idiopathic scoliosis. Spine. 1986; 11:779.

29. Goldberg CJ, Dowling FE, Hall JE, et al. A statistical comparison between natural history of idiopathic scoliosis and brace treatment in skeletally immature adolescent girls. Spine. 1993; 18:902-8. [PubMed: 8316891]

30. Goldberg CJ, Moore DP, Fogarty EE, et al. Adolescent idiopathic scoliosis: the effect of brace treatment on the incidence of surgery. Spine. 2001; 26:42-7. [PubMed: 11148644]

31. Green NE. Part-time bracing of adolescent idiopathic scoliosis. J Bone and Joint Surg. 1986; 68:738-42. [PubMed: 3722232]

32. Hanks GA, Zimmer B, Nogi J. TLSO treatment of idiopathic scoliosis. An analysis of the Wilmington jacket. Spine. 1988; 13:626-9. [PubMed: 3175752]

33. Howard A, Wright JG, Hedden D. A comparative study of TLSO, Charleston, and Milwaukee braces for idiopathic scoliosis. Spine. 1998; 23:2404-11. [PubMed: 9836354]

34. Janicki JA, Poe-Kochert C, Armstrong DG, et al. A comparison of the thoracolumbosacral orthoses and providence orthosis in the treatment of adolescent idiopathic scoliosis: results using the new SRS inclusion and assessment criteria for bracing studies. J Pediatr Orthop. 2007; 27:369-74. [PubMed: 17513954]

35. Jonasson-Rajala E, Josefsson E, Lundberg B, et al. Boston thoracic brace in the treatment of idiopathic scoliosis. Initial correction. Clinical Orthopaedics \& Related Research. 1984:37-41. [PubMed: 6697599]

36. Karol LA. Effectiveness of bracing in male patients with idiopathic scoliosis. Spine. 2001; 26:2001-5. [PubMed: 11547200]

37. Katz, DE.; Browne, RH. The influence of how much a scoliosis orthosis is worn and its ability to prevent curve progression in adolescent idiopathic scoliosis.. Scoliosis Research Society Annual Meeting.; Quebec City, Quebec. 2003;

38. Katz DE, Durrani AA. Factors that influence outcome in bracing large curves in patients with adolescent idiopathic scoliosis. Spine. 2001; 26:2354-61. [PubMed: 11679821]

39. Katz DE, Herring JA, Browne RH, et al. Brace wear control of curve progression in adolescent idiopathic scoliosis. J Bone and Joint Surg. 2010; 92:1343-52. [PubMed: 20516309]

40. Katz DE, Richards BS, Browne RH, et al. A comparison between the Boston brace and the Charleston bending brace in adolescent idiopathic scoliosis. Spine. 1997; 22:1302-12. [PubMed: 9201832]

41. Korovessis P, Kyrkos C, Piperos G, et al. Effects of thoracolumbosacral orthosis on spinal deformities, trunk asymmetry, and frontal lower rib cage in adolescent idiopathic scoliosis. Spine. 2000; 25:2064-71. [PubMed: 10954637]

42. Laurnen EL, Tupper JW, Mullen MP. The Boston brace in thoracic scoliosis. A preliminary report. Spine. 1983; 8:388-95. [PubMed: 6635788]

43. Lee CS, Hwang CJ, Kim DJ, et al. Effectiveness of the Charleston night-time bending brace in the treatment of adolescent idiopathic scoliosis. J Pediatr Orthop. 2012; 32:368-72. [PubMed: 22584837]

44. Lonstein JE, Winter RB. The Milwaukee brace for the treatment of adolescent idiopathic scoliosis. A review of one thousand and twenty patients. Journal of Bone \& Joint Surgery - American Volume. 1994; 76:1207-21.

45. Mellencamp DD, Blount WP, Anderson AJ. Milwaukee brace treatment of idiopathic scoliosis: late results. Clinical Orthopaedics \& Related Research. 1977:47-57. [PubMed: 598139]

46. Miller JA, Nachemson AL, Schultz AB. Effectiveness of braces in mild idiopathic scoliosis. Spine. 1984; 9:632-5. [PubMed: 6495034]

47. Moe JH, Kettleson DN. Idiopathic scoliosis. Analysis of curve patterns and the preliminary results of Milwaukee-brace treatment in one hundred sixty-nine patients. J Bone and Joint Surg. 1970; 52:1509-33. [PubMed: 5483076] 
48. Montgomery F, Willner S. Prognosis of brace-treated scoliosis. Comparison of the Boston and Milwaukee methods in 244 girls. Acta Orthopaedica Scandinavica. 1989; 60:383-5. [PubMed: 2816312]

49. Nachemson AL, Peterson LE. Effectiveness of treatment with a brace in girls who have adolescent idiopathic scoliosis. A prospective, controlled study based on data from the Brace Study of the Scoliosis Research Society. J Bone and Joint Surg. 1995; 77:815-22. [PubMed: 7782353]

50. Negrini S, Atanasio S, Fusco C, et al. Effectiveness of complete conservative treatment for adolescent idiopathic scoliosis (bracing and exercises) based on SOSORT management criteria: results according to the SRS criteria for bracing studies - SOSORT Award 2009 Winner. Scoliosis. 2009; 4:19. [PubMed: 19732429]

51. Negrini S, Minozzi S, Bettany-Saltikov J, et al. Braces for idiopathic scoliosis in adolescents. Spine (Phila Pa 1976). 2010; 35:1285-93. [PubMed: 20461027]

52. Noonan KJ, Weinstein SL, Jacobson WC, et al. Use of the Milwaukee brace for progressive idiopathic scoliosis. J Bone and Joint Surg. 1996; 78:557-67. [PubMed: 8609134]

53. Olafsson Y, Saraste H, Soderlund V, et al. Boston brace in the treatment of idiopathic scoliosis. Journal of Pediatric Orthopedics. 1995; 15:524-7. [PubMed: 7560048]

54. Peltonen J, Poussa M, Ylikoski M. Three-year results of bracing in scoliosis. Acta Orthopaedica Scandinavica. 1988; 59:487-90. [PubMed: 3188850]

55. Piazza MR, Bassett GS. Curve progression after treatment with the Wilmington brace for idiopathic scoliosis. Journal of Pediatric Orthopedics. 1990; 10:39-43. [PubMed: 2298893]

56. Price CT, Scott DS, Reed FE Jr. et al. Nighttime bracing for adolescent idiopathic scoliosis with the Charleston bending brace. Preliminary report. Spine. 1990; 15:1294-9. [PubMed: 2281373]

57. Price CT, Scott DS, Reed FR Jr. et al. Nighttime bracing for adolescent idiopathic scoliosis with the Charleston Bending Brace: long-term follow-up. Journal of Pediatric Orthopedics. 1997; 17:703-7. [PubMed: 9591969]

58. Rowe DE, Bernstein SM, Riddick MF, et al. A meta-analysis of the efficacy of non-operative treatments for idiopathic scoliosis. J Bone and Joint Surg. 1997; 79:664-74. [PubMed: 9160938]

59. Trivedi JM, Thomson JD. Results of Charleston bracing in skeletally immature patients with idiopathic scoliosis. Journal of Pediatric Orthopedics. 2001; 21:277-80. [PubMed: 11371804]

60. Upadhyay SS, Nelson IW, Ho EK, et al. New prognostic factors to predict the final outcome of brace treatment in adolescent idiopathic scoliosis. Spine. 1995; 20:537-45. [PubMed: 7604322]

61. Watts HG, Hall JE, Stanish W. The Boston brace system for the treatment of low thoracic and lumbar scoliosis by the use of a girdle without superstructure. Clinical Orthopaedics \& Related Research. 1977:87-92. [PubMed: 598144]

62. Wever DJ, Tonseth KA, Veldhuizen AG, et al. Curve progression and spinal growth in brace treated idiopathic scoliosis. Clinical Orthopaedics \& Related Research. 2000:169-79. [PubMed: 10943199]

63. Wiley JW, Thomson JD, Mitchell TM, et al. Effectiveness of the Boston brace in treatment of large curves in adolescent idiopathic scoliosis. Spine. 2000; 25:2326-32. [PubMed: 10984784]

64. Willers U, Normelli H, Aaro S, et al. Long-term results of Boston brace treatment on vertebral rotation in idiopathic scoliosis. Spine. 1993; 18:432-5. [PubMed: 8470002]

65. Willner S. Effect of the Boston thoracic brace on the frontal and sagittal curves of the spine. Acta Orthopaedica Scandinavica. 1984; 55:457-60. [PubMed: 6475514]

66. Wong MS, Cheng JC, Lam TP, et al. The effect of rigid versus flexible spinal orthosis on the clinical efficacy and acceptance of the patients with adolescent idiopathic scoliosis. Spine (Phila Pa 1976). 2008; 33:1360-5. [PubMed: 18496349]

67. Ylikoski M, Peltonen J, Poussa M. Biological factors and predictability of bracing in adolescent idiopathic scoliosis. Journal of Pediatric Orthopedics. 1989; 9:680-3. [PubMed: 2600176]

68. Yrjonen T, Ylikoski M, Schlenzka D, et al. Effectiveness of the Providence nighttime bracing in adolescent idiopathic scoliosis: a comparative study of 36 female patients. Eur Spine J. 2006; 15:1139-43. [PubMed: 16429287]

69. Zaborowska-Sapeta K, Kowalski IM, Kotwicki T, et al. Effectiveness of Cheneau brace treatment for idiopathic scoliosis: prospective study in 79 patients followed to skeletal maturity. Scoliosis. 2011; 6:2. [PubMed: 21266084] 
70. Dolan LA, Donnelly MJ, Spratt KF, et al. Professional opinion concerning the effectiveness of bracing relative to observation in adolescent idiopathic scoliosis. J Pediatr Orthop. 2007; 27:2706. [PubMed: 17414008]

71. Dolan LA, Sabesan V, Weinstein SL, et al. Preference assessment of recruitment into a randomized trial for adolescent idiopathic scoliosis. J Bone and Joint Surg. 2008; 90:2594-605. [PubMed: 19047704]

72. Dolan, LA.; Weinstein, SL.; Adams, BS. Temperature as a diagnostic test for compliance with a thoracolumbosacral orthosis.. Annual Meeting of the Pediatric Orthopaedic Society of North America; 2010;

73. Weinstein SL, Ponseti IV. Curve progression in idiopathic scoliosis. J. Bone and Joint. Surg. 1983; 65-A:447-55. [PubMed: 6833318]

74. Peterson LE, Nachemson AL. Prediction of progression of the curve in girls who have adolescent idiopathic scoliosis of moderate severity. Logistic regression analysis based on data from The Brace Study of the Scoliosis Research Society. J Bone and Joint Surg. 1995; 77:823-7. [PubMed: 7782354]

75. Lonstein JE, Carlson JM. The prediction of curve progression in untreated idiopathic scoliosis during growth. J Bone and Joint Surg. 1984; 66:1061-71. [PubMed: 6480635]

76. Landgraf, J.; Abetz, L.; Ware, J. The CHQ User's Manual. The Health Institute, New England Medical Center; Boston, MA: 1996.

77. Varni J, Seid M, Rode C. The PedsQL: Measurement model for the pediatric quality of life inventory. Medical Care. 1999; 37:126-39. [PubMed: 10024117]

78. Petersen A, Schulenberg J, Abramowitz R, et al. A self-image questionnaire for young adolescents (SIQYA): Reliability and validity. J Youth Adol. 1984; 13:93-111.

79. Sanders JO, Harrast JJ, Kuklo TR, et al. The Spinal Appearance Questionnaire: results of reliability, validity, and responsiveness testing in patients with idiopathic scoliosis. Spine. 2007; 32:2719-22. [PubMed: 18007251]

80. Ylikoski M. Growth and progression of adolescent idiopathic scoliosis in girls. J Pediatr Orthop B. 2005; 14:320-4. [PubMed: 16093941]

81. Sanders JO, Browne RH, McConnell SJ, et al. Maturity assessment and curve progression in girls with idiopathic scoliosis. J Bone and Joint Surg. 2007; 89:64-73. [PubMed: 17200312]

82. Sanders JO, Khoury JG, Kishan S, et al. Predicting scoliosis progression from skeletal maturity: a simplified classification during adolescence. J Bone Joint Surg Am. 2008; 90:540-53. [PubMed: 18310704]

83. Rubin DB. For objective causal inference, design trumps analysis. Annals of Applied Statistics. 2008; 2:808-40.

84. D'Agostino RB Jr. Propensity score methods for bias reduction in the comparison of a treatment to a non-randomized control group. Stat Med. 1998; 17:2265-81. [PubMed: 9802183]

85. Lan KKG, DeMets DL. Changing frequency of interim analysis in sequential monitoring. Biometrics. 1989; 45:1017-20. [PubMed: 2790114]

86. O'Brien PC, Fleming TR. A multiple testing procedure for clinical trials. Biometrics. 1979

87. O'Brien, MF.; Kuklo, TR.; Blanke, KM., et al. Radiographic Measurement Manual. Spinal Study Deformity Group. , editor. Medtronic Sofamor Danek USA, Inc.; 2004.

88. Nash CI, Moe JH. A study of vertebral rotation. J Bone Joint Surg AM. 1969; 2:223-9. [PubMed: 5767315] 


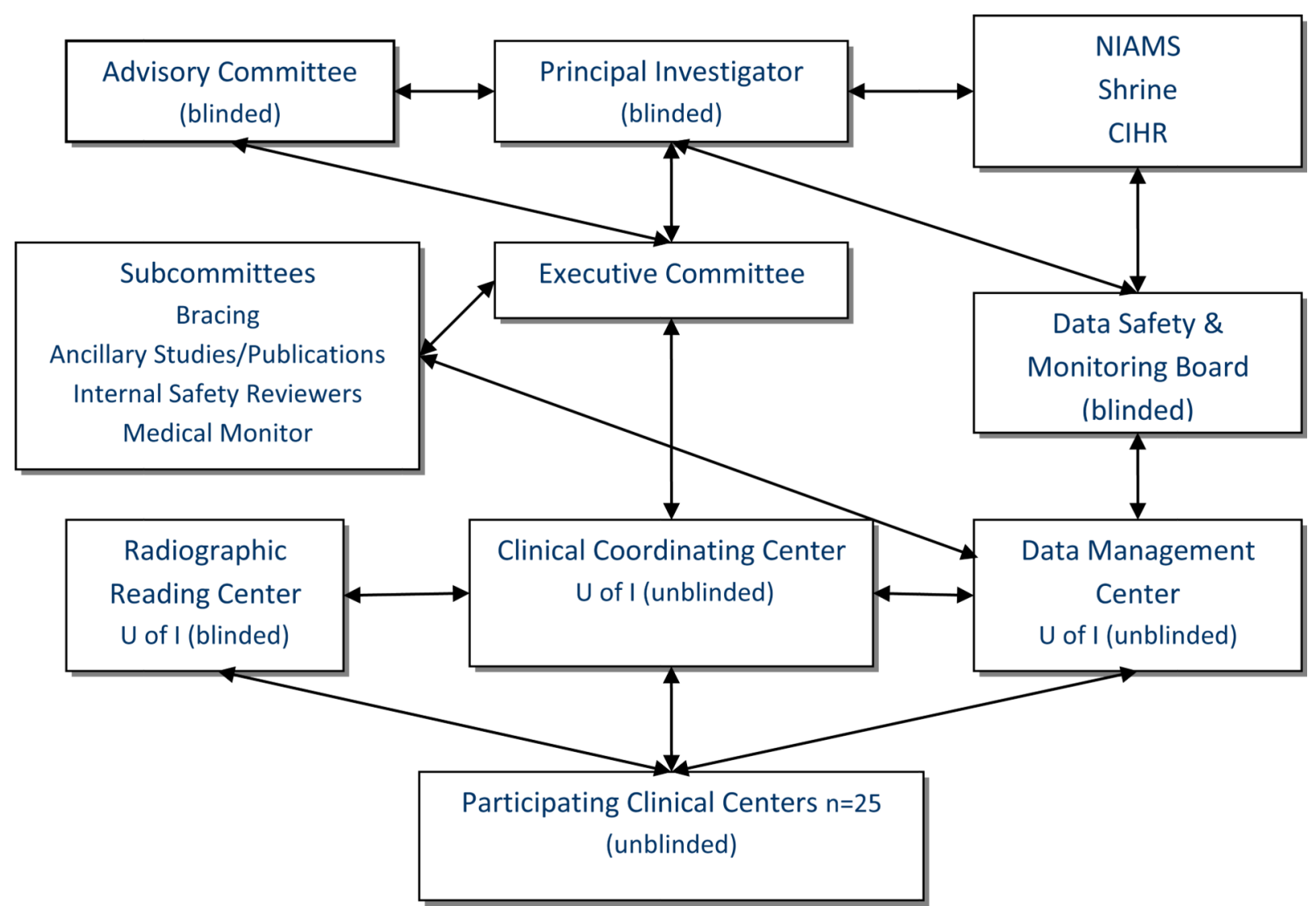

Figure 1.

Organizational Structure of BrAIST 


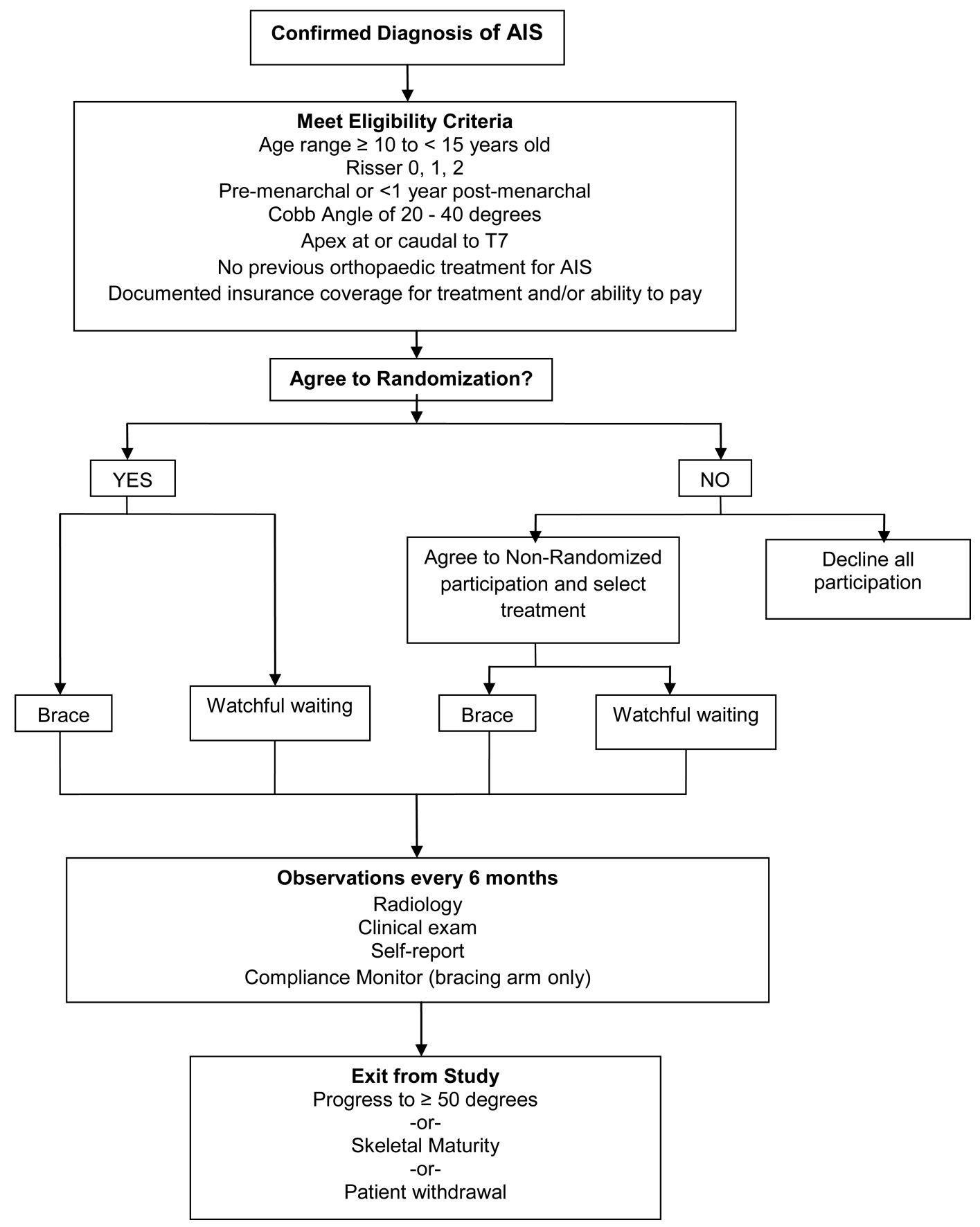

Figure 2.

Subject Flow Chart 


\section{Table I}

\section{Participating Centers by Funding Source}

\begin{tabular}{|c|c|}
\hline \multicolumn{2}{|l|}{ NIH (n=15) } \\
\hline University of Virginia & University of New Mexico \\
\hline Washington University in St. Louis & Children's Hospital of Boston \\
\hline Children's Hospital of Central California & Cincinnati Children's Hospital Medical Center \\
\hline Rady Children's Hospital & Children's Orthopaedics of Atlanta \\
\hline Nemours/ DuPont Hospital for Children & Seattle Children's Hospital \\
\hline University of Iowa & Children's Hospital of Philadelphia \\
\hline Children's Hospital of Pittsburgh & Hospital for Special Surgery \\
\hline \multicolumn{2}{|l|}{ Mercy Children's Hospital Kansas City ${ }^{*}$} \\
\hline \multicolumn{2}{|l|}{ Shriners Hospitals for Children (n=7) } \\
\hline Montreal & St. Louis \\
\hline Salt Lake City & Lexington \\
\hline Chicago & Sacramento \\
\hline \multicolumn{2}{|l|}{ Twin Cities } \\
\hline \multicolumn{2}{|c|}{ Canadian Institutes of Health Research $(n=2)$} \\
\hline Hospital for Sick Children, Toronto & University of British Columbia \\
\hline \multicolumn{2}{|l|}{ Internally-Funded (n=1) } \\
\hline University of Rochester & \\
\hline
\end{tabular}




\section{Table II}

\section{Inclusion Criteria}

\section{Diagnosis of AIS}

Presenting without associated musculoskeletal, neurological or other conditions possibly responsible for the curvature

Maturity

Age between 10 and 15 at consent

Risser 0,1 , or 2

Girls: Pre-menarchal OR post-menarchal by less than 1 year

Curve Magnitude

Largest Cobb angle between 20 and 39 degrees

Cobb angle $<20$ degrees until the age of 10

Treatment

No history of previous surgical or orthotic treatment

Physical and mental ability to adhere to bracing protocol

Ability to read and write English, Spanish or French 
Table III

Data Sources and Collection Schedule

\begin{tabular}{|c|c|c|c|c|}
\hline & Enrollment & In-Brace film & Q6 mo & Yearly \\
\hline Radiographic Data & PA, lateral, bending, hand & PA & PA, hand & PA, lateral, hand \\
\hline Cobb angle of structural and compensatory curves ${ }^{110}$ & $\mathrm{P}, \mathrm{R}$ & $\mathrm{R}$ & $\mathrm{P}, \mathrm{R}$ & $\mathrm{P}, \mathrm{R}$ \\
\hline Apex and end vertebrae of curves ${ }^{110}$ & $\mathrm{P}, \mathrm{R}$ & $\mathrm{R}$ & $\mathrm{P}, \mathrm{R}$ & $\mathrm{P}, \mathrm{R}$ \\
\hline Risser grade ${ }^{110}$ & $\mathrm{P}, \mathrm{R}$ & & $\mathrm{P}, \mathrm{R}$ & $\mathrm{P}, \mathrm{R}$ \\
\hline Nash Moe rotation ${ }^{111}$ & $\mathrm{R}$ & & $\mathrm{R}$ & $\mathrm{R}$ \\
\hline Percent curve correction & $\mathrm{R}$ & $\mathrm{R}$ & & \\
\hline Sagittal balance ${ }^{110}$ & $\mathrm{R}$ & & & $\mathrm{R}$ \\
\hline Coronal balance ${ }^{110}$ & $\mathrm{R}$ & $\mathrm{R}$ & $\mathrm{R}$ & $\mathrm{R}$ \\
\hline \multicolumn{5}{|l|}{ Kyphosis ${ }^{110}$} \\
\hline \multicolumn{5}{|l|}{ Lordosis ${ }^{110}$} \\
\hline Spinal length $\mathrm{T}_{1}-\mathrm{L}_{4}{ }^{57}$ & $\mathrm{R}$ & & $\mathrm{R}$ & $\mathrm{R}$ \\
\hline Concave-to-convex vertebral height ratio ${ }^{17}$ & $\mathrm{R}$ & & $\mathrm{R}$ & $\mathrm{R}$ \\
\hline Digital maturity stage ${ }^{81}$ & $\mathrm{R}$ & & $\mathrm{R}$ & $\mathrm{R}$ \\
\hline \multicolumn{5}{|l|}{ Clinical Data } \\
\hline Weight, sitting and standing height & $\mathrm{P}$ & & $P$ & $\mathrm{P}$ \\
\hline Ortho/neuro examination & $\mathrm{P}$ & & $P$ & $\mathrm{P}$ \\
\hline \multicolumn{5}{|l|}{ Orthotic Data (brace arm only) } \\
\hline Dose logger data & & & $P$ & $\mathrm{P}$ \\
\hline Brace wear diary & & & S & $\mathrm{S}$ \\
\hline Skin condition under brace & & & $\mathrm{P}$ & $\mathrm{P}$ \\
\hline Orthotist clinical notes & $\mathrm{P}$ & $\mathrm{P}$ & $P$ & $\mathrm{P}$ \\
\hline Brace Quality Evaluation & & $\mathrm{BEC}$ & & \\
\hline \multicolumn{5}{|l|}{ Self-Report Data } \\
\hline Child Health Questionnaire ${ }^{83}$ & $\mathrm{~S}$ & & S & $\mathrm{S}$ \\
\hline Self-Image Questionnaire for Young & $\mathrm{S}$ & & S & $\mathrm{S}$ \\
\hline \multicolumn{5}{|l|}{ Adolescents ${ }^{85}$} \\
\hline PedsQL ${ }^{84}$ & $\mathrm{~S}$ & & $\mathrm{~S}$ & $\mathrm{~S}$ \\
\hline \multicolumn{5}{|l|}{ Spinal Appearance Questionnaire ${ }^{112}$} \\
\hline Demographic information & $\mathrm{S}$ & & S & $\mathrm{S}$ \\
\hline Menarchal status & $\mathrm{S}$ & & $\mathrm{S}$ & $\mathrm{S}$ \\
\hline
\end{tabular}

Key: P=Participating Clinical Centers; R = Radiographic Reading Center; $\mathrm{S}=$ Self Report; BEC=Bracing Evaluation Committee 
Table IV

Tolerance Limits for Radiographic Evaluations

\begin{tabular}{|lll|}
\hline Evaluation & Tolerance Limit \\
\hline Cobb angle & $0-5$ degrees \\
\hline Risser grade & Within range of 0, 1,2, or 3, 4, 5 \\
\hline Rotation (Nash Moe) & Within range of 0, 1,2, or 3, & \\
\hline Kyphosis & Normal & $10-40$ degrees \\
& Hypokyphotic & $<10$ degrees \\
& Hyperkyphotic & $>40$ degrees \\
\hline Lordosis & Normal & $40-60$ degrees \\
& Hypokyphotic & $<40$ degrees \\
& Hyperkyphotic & $>60$ degrees \\
\hline Digital Maturity Stage & Agree on stages 2 and 3; in cases other than the endpoint \\
\hline
\end{tabular}


Table V

Sample Size and Power Calculations

\begin{tabular}{|c|c|}
\hline Control Failure Rate & $30 \%$ \\
\hline Bracing Failure Rate & $15 \%$ \\
\hline Significance Level & 0.05 \\
\hline Sample Size & 348 \\
\hline Sample Size Adjusted for $10 \%$ drop-out & 384 \\
\hline Percent of subjects in braced group & Power \\
\hline $25 \%$ & 0.82 \\
\hline $40 \%$ & 0.91 \\
\hline $\mathbf{5 0 \%}$ & 0.92 \\
\hline $60 \%$ & 0.91 \\
\hline $75 \%$ & 0.85 \\
\hline
\end{tabular}

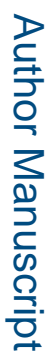

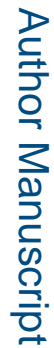

